\title{
ORGANIZACIONES JUSTAS: ¿ES POSIBLE CONSTRUIRLAS?
}

FAIR ORGANIZATIONS: IS IT POSSIBLE TO BUILD THEM?

\author{
Elsa Maria Vasquez Trespalacios \\ Universidad de Guadalajara \\ Guadalajara, México \\ evasquez@ces.edu.co
}

\author{
Carolina Aranda Beltran \\ Universidad de Guadalajara \\ Guadalajara, México \\ caranda2000@yahoo.com.mx
}

Fecha de recepción: 17/12/2019 - Fecha de aprobación: 09/03/2020
DOI: https://doi.org/10.36995/j.visiondefuturo.2020.24.02.005.es

\section{RESUMEN}

El bienestar de los trabajadores y la productividad de las empresas han sido temas de gran interés en la literatura científica y en la vida diaria de las organizaciones. La justicia organizacional es uno de los factores que contribuye a que se alcancen estos resultados.

Las teorías que intentan explicar los efectos que tiene una percepción de justicia en el trabajo hacen énfasis en la relación de reciprocidad que debe existir entre el empleado y el empleador. La percepción de que el empleador actúa de forma justa da origen a sentimientos de confianza, compromiso y lealtad hacia el empleador, lo que repercute en un mejor desempeño laboral y en unas actitudes positivas en el lugar de trabajo.

Cada uno de los procedimientos que se llevan a cabo en el ambiente laboral, tales como la selección de personal, la evaluación de desempeño, la resolución de conflictos, la distribución de las recompensas y los despidos tienen un alto riesgo de generar percepciones de injusticia por las partes involucradas, es por esto que deben repensarse a la luz de las dimensiones de la justicia organizacional.

El objetivo entonces es conocer de qué forma se puede aplicar el concepto de la justicia en los procedimientos más importantes a nivel organizacional.

PALABRAS CLAVE: Justicia Organizacional; Satisfacción Laboral; Procedimientos; Comunicación; Despidos.

\section{ABSTRACT}

The well-being of workers and the productivity of companies have been subjects of great interest in the scientific literature and in the daily life of organizations. Organizational justice is one of the factors that contributes to achieving these results.

Theories that attempt to explain the effects of a perception of justice at work emphasize the relationship of reciprocity that must exist between the employee and the employer. The perception that the employer acts fairly gives rise to feelings of trust, commitment and loyalty "Visión de Futuro" Año 17, Volumen N²4 N², Julio - Diciembre 2020 - Pág 166 - 183 URL de la Revista: http://visiondefuturo.fce.unam.edu.ar/index.php/visiondefuturo/index URL del Documento: https://visiondefuturo.fce.unam.edu.ar/index.php/visiondefuturo/issue/view/18 
to the employer, which has an impact on better job performance and positive attitudes in the workplace.

Each of the procedures carried out in the work environment, such as the selection of personnel, performance evaluation, conflict resolution, distribution of rewards and layoffs, are at high risk of generating perceptions of injustice due to the parties involved, this is why they must be rethought in the light of the dimensions of organizational justice.

The objective then is to know how the concept of justice can be applied in the most important procedures at the organizational level

KEYWORDS: Organizational Justice; Job Satisfaction; Procedures; Communication; Layoffs.

\section{INTRODUCCIÓN}

La Justicia organizacional como concepto tuvo sus inicios dentro de las ciencias sociales y económicas. Actualmente existe un interés creciente en su estudio y en relacionarla con algunos eventos relevantes a las organizaciones, tales como el compromiso, la satisfacción laboral, la confianza y el desempeño (Khiavi, Shakhi, Dehghani, \& Zahiri, 2016; Vaamonde, Omar, \& Salessi, 2018; Wan, Zhou, Li, \& Shang, 2018).

Teóricamente, en la literatura concerniente a la justicia organizacional, se argumenta que las personas buscan identidades sociales positivas, por lo tanto, al observar condiciones justas en su lugar de trabajo pueden sentir y reconocer su status dentro del grupo (Blader \& Tyler, 2009; Tyler \& Blader, 2000). Debido a esta relación entre la persona y su lugar de trabajo, se puede considerar que la injusticia organizacional actúa como un estresor social ya que evidencia una relación adversa al excluir a la persona de su grupo social (Denise Francis, 2019; Dishon-Berkovits, 2017).

La percepción de injusticia en el lugar de trabajo ha sido ligada de manera consistente con varios comportamientos relacionados con el trabajo, tales como el compromiso, la intención de abandonar la organización, la motivación y la productividad, así como con consecuencias negativas para la salud física y mental (Herr et al., 2016; Loerbroks et al., 2014).

Cuando se percibe injusticia, el trabajador siente que sus aportes no son valorados adecuadamente y que se dificulta la consecución de recursos, lo que repercute en forma directa sobre su bienestar, reduciendo su compromiso con el trabajo y aumentando la presencia de eventos relacionados con una exposición crónica al estrés, como el síndrome de burnout.

\footnotetext{
"Visión de Futuro" Año 17, Volumen N²4 N², Julio - Diciembre 2020 - Pág 166 - 183

URL de la Revista: http://visiondefuturo.fce.unam.edu.ar/index.php/visiondefuturo/index

URL del Documento: https://visiondefuturo.fce.unam.edu.ar/index.php/visiondefuturo/issue/view/18

ISSN 1668 - 8708 - Versión en Línea

E-mail: revistacientifica@fce.unam.edu.ar
} 
Los comportamientos contraproducentes en el trabajo, definidos como aquellos que tienen como finalidad hacer daño a la organización o a los individuos dentro de ella, tales como el sabotaje, el uso indebido de los bienes, fraude, robo, retirarse antes del trabajo, desperdicio de recursos, etc. se han relacionado también con la percepción de injusticia organizacional por parte de los trabajadores (Schulte-Braucks, Baethge, Dormann, \& VahleHinz, 2019).

Es por esto que es de gran interés para las organizaciones alinear sus procesos según la visión de justicia organizacional. La presente revisión aborda la operacionalización de las dimensiones de la justicia en los procesos organizacionales y plantea las posibilidades de investigación en este tema.

\section{Metodología}

Se realizó una búsqueda bibliográfica de artículos científicos originales publicados en idiomas inglés y español, en revistas revisadas por pares, sin límite de tiempo. El proceso de búsqueda de la información incluyó las referencias bibliográficas contenidas en las bases de datos: Pubmed, Ebsco, Lilacs, Scopus y Web of Science. Los términos de búsqueda utilizados fueron: Organizational justice, Procedural justice, Distributive justice, Interactional justice, Personnel selection, Layoffs, Punishment, Conflict resolution, Performance appraisal todos ellos identificados previamente como términos Mesh o sus equivalentes DecS en español, y se realizó una búsqueda adicional mediante la revisión de las referencias bibliográficas de los artículos identificados. Inicialmente, los artículos fueron identificados a través de la búsqueda individual de cada una de las investigadoras, y fueron analizados separadamente. Las discrepancias se resolvieron mediante el concepto de un tercer evaluador.

\section{DESARROLLO}

La justicia organizacional expresa el grado al cual los individuos creen que los resultados que reciben y la forma como son tratados dentro de una organización son justos, equitativos y se encuentran en sincronía con los principios morales y éticos (Cropanzano \& Ambrose, 2001).

La clasificación más popular de las dimensiones del constructo de justicia organizacional son la justicia distributiva y la justicia de los procedimientos o procedimental (Cropanzano R \& Folger R, 1991), sin embargo, alrededor de 1986, fue introducida una nueva dimensión, llamada justicia de las interacciones (Bies \& Moag, 1986). Un estudio posterior postuló que la justicia de las interacciones o interaccional debía ser divida en dos

\footnotetext{
"Visión de Futuro" Año 17, Volumen N$^{0} 24$ N² 2, Julio - Diciembre 2020 - Pág 166 - 183

URL de la Revista: http://visiondefuturo.fce.unam.edu.ar/index.php/visiondefuturo/index

URL del Documento: https://visiondefuturo.fce.unam.edu.ar/index.php/visiondefuturo/issue/view/18

ISSN 1668 - 8708 - Versión en Línea

E-mail: revistacientifica@fce.unam.edu.ar
} 
con el fin de ampliar su capacidad discriminante: justicia interpersonal, entendida como la imparcialidad en el comportamiento interpersonal visto durante la toma de decisiones sobre los procedimientos y distribución de los resultados y justicia informacional, referida a la imparcialidad en términos de las explicaciones e información proporcionada (Greenberg, 1993). A continuación, se describe cada una de las dimensiones:

\section{Justicia distributiva}

Un pilar esencial para la comprensión de la justicia distributiva es proporcionada por la teoría de la equidad, postulada por Adams (1965), en donde se establece una relación entre aportes que pueden ser de diversos tipos (esfuerzo, experiencia laboral, dedicación, edad, etc.) y algunos resultados que pueden ser positivos (salario, beneficios, recompensas intrínsecas al trabajo) o negativos (fatiga, monotonía, condiciones laborales pobres, incertidumbre, violencia, etc.), en este sentido, las personas realizan una comparación de sus aportes y resultados con otras personas dentro y fuera de la organización y analizan la imparcialidad de esta observación mediante un cociente. Sí después de realizada la comparación, el individuo percibe que es imparcial, entonces se puede decir que existe justicia distributiva. Pero cuando la percepción es de injusticia o imparcialidad se puede llegar a estados de ansiedad, distrés, sentirse poco compensado (resentimiento) o sobrecompensado (culpa).

\section{Justicia procedimental}

Es la imparcialidad sentida por los miembros de una organización en el proceso de toma de decisiones (Leventhal, Karuza, \& Fry, 1980). Los procedimientos se consideran justos cuando fomentan resultados justos. El aspecto crítico de esta dimensión son los medios por los cuales se alcanzan los resultados (Folger \& Greenberg, 1985).

Sí los procedimientos dentro de una organización son vistos como justos, el pensamiento que tiene un individuo de su organización será influenciado positivamente, lo que lleva a mayor lealtad y mayores deseos de trabajar en la organización. A los estudios de Leventhal (1980) se le atribuye que el estudio de la justicia de los procedimientos se haya expandido de los ámbitos meramente legales a los ámbitos laborales.

Para que un proceso o procedimiento según Leventhal (1980), sea considerado justo, debe cumplir los siguientes criterios:

- Ser practicado consistentemente a través del tiempo y de los individuos

- Estar absolutamente libre de favoritismo

- Poseer mecanismos para corregir las decisiones erradas o inexactas

\footnotetext{
"Visión de Futuro" Año 17, Volumen No 24 N² 2, Julio - Diciembre 2020 - Pág 166 - 183

URL de la Revista: http://visiondefuturo.fce.unam.edu.ar/index.php/visiondefuturo/index

URL del Documento: https://visiondefuturo.fce.unam.edu.ar/index.php/visiondefuturo/issue/view/18

ISSN 1668 - 8708 - Versión en Línea

E-mail: revistacientifica@fce.unam.edu.ar
} 
- Aceptar y cumplir con estándares éticos y morales

- Asegurarse de que los múltiples grupos afectados por una decisión sean escuchados

\section{Justicia interaccional}

Bies y Moag (1986) al introducir el concepto de justicia de las interacciones, afirman que junto con la evaluación de los procesos y los resultados, los empleados también evalúan la forma como son tratados. La teoría del intercambio social y el principio de la reciprocidad son las bases sobre las que se establece la justicia interaccional dentro de las organizaciones (Cropanzano \& Mitchell, 2005).

Como se mencionó anteriormente, la justicia interaccional fue dividida en dos: justicia interpersonal: entendida como el grado en que una persona de autoridad trata a las demás personas con respeto y dignidad mientras implementa procesos y procedimientos organizacionales y justicia de la información que se refiere a las explicaciones que se brindan a los empleados sobre el porqué ciertos procedimientos fueron aplicados de determinada forma o porqué los resultados fueron distribuidos de alguna manera (Greenberg, 1990) en otras palabras, trata con la veracidad y justificación de la información que se da a los empleados. Esto debido a que las percepciones de que la información es inadecuada o falsa llevan a la visión de injusticia.

Para delinear de una manera clara el concepto de justicia interaccional, Bies y Moag (1986), enuncian los cuatro conceptos que la definen:

- Respeto: seriedad y dignidad para tratar a los subordinados

- Propiedad: evitar afirmaciones imprudentes y perjudiciales

- Justificación: las razones inherentes a una decisión deben ser explicadas claramente

- Veracidad: las razones dadas deben ser veraces, francas y honestas

Todos los tres tipos de justicia funcionan en interrelación, sin embargo, cuando se desea promover la justicia en el lugar de trabajo, deben ser analizadas separadamente.

\section{Operacionalización del concepto de Justicia Organizacional Proceso de selección de personal}

Dentro del contexto organizacional, el proceso de selección de personal es quizá uno de los que mayor incertidumbre lleva consigo, debido a que los aplicantes a un proceso no conocen cuáles son sus probabilidades de tener éxito en el mismo y poseen poca información sobre cómo será trabajar para un determinado empleador. Es por esto que se argumenta desde el punto de vista teórico que en la selección de personal son

\footnotetext{
"Visión de Futuro" Año 17, Volumen No 24 N², Julio - Diciembre 2020 - Pág 166 - 183

URL de la Revista: http://visiondefuturo.fce.unam.edu.ar/index.php/visiondefuturo/index

URL del Documento: https://visiondefuturo.fce.unam.edu.ar/index.php/visiondefuturo/issue/view/18

ISSN 1668 - 8708 - Versión en Línea

E-mail: revistacientifica@fce.unam.edu.ar
} 
fundamentales las percepciones de justicia (Truxillo, Bauer, \& Garcia, 2017; van den Bos \& Lind, 2002).

El estudio de las percepciones de justicia sobre los procesos de selección de personal, se ha llevado a cabo tradicionalmente solicitando a los empleados que evalúen sí una técnica o procedimiento de selección es justo para contratar aplicantes. Una revisión al respecto muestra que existe mucha variabilidad en la percepción de justicia de diferentes técnicas, en términos generales, las entrevistas, hojas de vida y simulaciones de realizar el trabajo, fueron evaluadas positivamente, mientras que el análisis de un manuscrito o los contactos personales del aplicante fueron evaluados negativamente (Konradt, Garbers, Weber, Erdogan, \& Bauer, 2015; McCarthy et al., 2017; Nikolaou \& Georgiou, 2018).

La operacionalización del concepto de justicia debe comenzar desde el mismo proceso de selección de los aspirantes. El uso de una rúbrica de evaluación, pruebas estandarizadas y un proceso de entrevistas que pueda ser realizado por al menos un miembro independiente de la organización pueden aumentar la percepción de justicia del futuro trabajador. Desde el punto de vista comunicativo, se debe explicar al aspirante cómo funciona el proceso de selección y cuál es el perfil esperado para el candidato a ocupar la posición vacante (Giannantonio Cristina, 2019; Konradt et al., 2015; McCarthy et al., 2017), estas explicaciones se ha visto que aumentan la percepción de justicia procedimental (Melchers \& Körner, 2019), sin embargo, en la literatura científica se expresa que aún falta investigación sobre los mensajes, el contenido y las consecuencias de presentar esta información a las personas interesadas en un cargo (Cortini, Galanti, \& Barattucci, 2019).

\section{Evaluación del desempeño}

Uno de los procesos vitales en el manejo de los recursos humanos de una empresa es la evaluación de desempeño de sus colaboradores, lo que permite posteriormente realizar la distribución de las recompensas a los mismos, hacer seguimiento del desarrollo laboral y en una última instancia tomar decisiones acerca de la permanencia de un empleado dentro de una organización. Con respecto a las evaluaciones de desempeño existe bastante insatisfacción por parte de los empleados, pues se manifiesta no estar de acuerdo con los procedimientos utilizados, ni con el tipo de retroalimentación recibida (Dusterhoff, Cunningham, \& Macgregor, 2014; Meinecke, Lehmann-Willenbrock, \& Kauffeld, 2017). Cuando un empleado cree que la evaluación de su desempeño tuvo un resultado negativo debido a condiciones como el género, la raza, la edad, alguna situación de discapacidad, puede aducir algún tipo de discriminación en este proceso (Rubin \& Edwards, 2018), también cuando considera que el trato dado por la persona que realiza la evaluación es sesgado (Mulders, 2019; Rubin \& Edwards, 2018; Tziner \& Levy, 2017).

\footnotetext{
"Visión de Futuro" Año 17, Volumen $N^{0} 24$ N² 2, Julio - Diciembre 2020 - Pág 166 - 183

URL de la Revista: http://visiondefuturo.fce.unam.edu.ar/index.php/visiondefuturo/index

URL del Documento: https://visiondefuturo.fce.unam.edu.ar/index.php/visiondefuturo/issue/view/18

ISSN 1668 - 8708 - Versión en Línea

E-mail: revistacientifica@fce.unam.edu.ar
} 
En este sentido, la aplicación del concepto de justicia a la evaluación de desempeño comprende una mayor participación del empleado, unas expectativas claras de desempeño desde la organización y los supervisores y un proceso comunicativo en donde se haga la retroalimentación de la evaluación al empleado (Ibeogu \& Ozturen, 2015). En última instancia, la aceptación del proceso de evaluación de desempeño depende de la percepción de que el procedimiento por el cual se lleva a cabo es justo. Otra sugerencia para mejorar esta percepción, se propone que el instrumento de evaluación de desempeño se ajuste para medir específicamente lo que se espera que un empleado realice en su trabajo (DeNisi \& Murphy, 2017).

La participación de los empleados en los diferentes momentos de la evaluación de desempeño logra que para ellos sea más fácil comprender los aspectos que la organización tendrá en cuenta para realizar la valoración y que se sientan más confiados de poder lograr lo que se espera de ellos. De la misma forma, una estrategia para aumentar la percepción de justicia en las evaluaciones de desempeño es el desarrollo de estrategias gerenciales para entrenar a quienes realizan este proceso (Babagana SA, 2019).

\section{Distribución de las recompensas}

El proceso de distribución de las recompensas en el lugar de trabajo es una de las principales herramientas para mantener e incrementar la satisfacción laboral de los empleados.

Tradicionalmente se han utilizado tres criterios para distribuir las recompensas laborales; la equidad, la igualdad y la necesidad (Cropanzano, Bowen, \& Gilliland, 2007; Fischer, 2008). Las distribuciones equitativas se realizan de acuerdo a las contribuciones particulares de cada empleado. Las distribuciones igualitarias establecen que cada uno de los empleados debe tener igualdad de oportunidades de alcanzar las recompensas, sin que sus características personales tengan influencia en esta distribución. Las distribuciones por necesidad se realizan según el requerimiento de cada individuo, por ejemplo, si el empleado tiene un nivel socioeconómico más bajo que otro, debe recibir mayor salario.

Las distribuciones equitativas de las recompensas a pesar de reconocer el esfuerzo individual, pueden favorecer la desigualdad, disminuyendo la satisfacción y colaboración de los demás trabajadores, tal como lo observaron Pfeffer y Langton (1993) en un estudio con personal académico, sin embargo, otro estudio realizado en el sector salud, no encontró relación entre un esquema de pago ligado al desempeño con la satisfacción laboral en el primer y cuarto año luego de la introducción de este modelo de recompensa (Allen, Whittaker, \& Sutton, 2017).

\footnotetext{
"Visión de Futuro" Año 17, Volumen N$^{0} 24$ N² 2, Julio - Diciembre 2020 - Pág 166 - 183

URL de la Revista: http://visiondefuturo.fce.unam.edu.ar/index.php/visiondefuturo/index

URL del Documento: https://visiondefuturo.fce.unam.edu.ar/index.php/visiondefuturo/issue/view/18

ISSN 1668 - 8708 - Versión en Línea

E-mail: revistacientifica@fce.unam.edu.ar
} 
Organizacionalmente, las recompensas tienen dos objetivos principales: motivar el desempeño individual y mantener la cohesión del grupo (Cropanzano et al., 2007), es por esto que los empleados de alto desempeño o aquellos con habilidades poco frecuentes de encontrar en el mercado, a la luz de la equidad, deberían tener mayores salarios y otro tipo de incentivos (Laundon, Cathcart, \& McDonald, 2019). En este punto, la justicia interaccional cobra una gran importancia, puesto que es a través de un proceso comunicativo en donde quedan explícitas las diferencias salariales y de otro tipo de recompensas que se otorgan en el ámbito organizacional, pudiendo así establecer que la justicia de la información tiene un papel mediador entre algunas recompensas y la satisfacción laboral (Gangloff, Mayoral, Personnaz, \& Rezazi, 2016; Long, 2016).

\section{Resolución de conflictos}

Los conflictos en el lugar de trabajo, definidos en un estudio observacional como eventos de alta tensión (Saxton, 2012), ocurren en una frecuencia que se ha estimado entre una y cuatro veces por procedimiento (Booij, 2007; Saxton, 2012) y aunque pudieran tener efectos positivos, tales como el mejor entendimiento de la tarea, del funcionamiento del equipo y toma de decisiones de mejor calidad (Greer, Saygi, Aaldering, \& de Dreu, 2012; Janss, Rispens, Segers, \& Jehn, 2012), no hay que desconocer también sus efectos negativos como la distracción de la tarea, y los costos para la organización. Teniendo en cuenta la inevitabilidad de las situaciones de conflicto, es necesario desarrollar habilidades para manejarlo.

Como causas frecuentes de conflicto en el lugar de trabajo, se han descrito la falta de claridad acerca de los objetivos o lineamientos, diferencias personales, conflictos de interés y los cambios dentro de la organización (Greer et al., 2012; Umiker W, 2005).

Algunos autores han propuesto diferentes estilos para el manejo del conflicto; según Thomas and Kilmann (1976), existen cinco estrategias de manejo: acomodación, evitación, colaboración, competencia y compromiso, dependiendo estos estilos no solo de la cultura de la organización sino del lugar geográfico en donde se presentan (Silva \& Caetano, 2016), sugieren que en el ámbito organizacional, se debe evitar el conflicto afectivo, es decir, aquel que ocurre cuando dos o más personas se dan cuenta de que tienen sentimientos y emociones irreconciliables, no obstante, el conflicto sustantivo, es decir, el que tienen dos o más personas con respecto a la tarea y su contenido, se debe mantener en forma moderada, dados los posibles efectos benéficos que esto tiene para la organización.

Los estilos negociados de resolución de conflictos, que a primera vista pudieran parecer los más equitativos, se han relacionado positivamente con una mayor carga psicológica, debido a que es difícil llegar a un acuerdo en un disputa en la cual cada parte 
está considerando solo sus intereses (Abas, Otto, \& Ramayah, 2018). Sin embargo, cuando este estilo de resolución de conflictos deba ser usado, es importante tener en cuenta que la justicia interaccional percibida puede actuar como moderadora de la carga somática y los síntomas depresivos de los trabajadores envueltos en situación de conflicto, sin embargo, se argumenta que aún faltan estudios al respecto que brinden un panorama más amplio (Sahoo, 2019).

Cuando una de las partes involucradas en conflicto es intransigente, la resolución del mismo se torna más difícil. En este punto encargado de recursos humanos o el supervisor deben realizar el arbitramiento en las situaciones de desacuerdo, es decir, actuar autocráticamente, es decir, decidir de qué forma se resolverá el conflicto. A pesar de que este arbitramiento conlleva el riesgo de no estar aprobado por alguna o todas las partes involucradas, sí este considera alguna de las dimensiones de la justicia organizacional, tiene una mayor probabilidad de ser aceptado (Goldman, 2003).

Despidos, situaciones de recorte de personal o renegociación de las condiciones de empleo/trabajo:

Los despidos pueden tener su origen en varias situaciones que ocurren en el ambiente de trabajo, una de ellas es el recorte o reducción de la plantilla; lo que comprende una serie de actividades ejecutadas intencionalmente por la gerencia para mejorar la eficiencia, afectando el tamaño de la empresa y sus procesos de trabajo (Cameron \& Freeman, 1996). Estas reducciones de plantilla pueden ocurrir de forma reactiva o proactiva para contener costos y aumentar la eficiencia, esto se logra eliminando o modificando el proceso productivo de la compañía.

Los despidos o recortes de personal son circunstancias de gran complejidad en el ámbito laboral, puesto que afectan tanto a los trabajadores que abandonan como a los que permanecen, aumentando la percepción de injusticia distributiva (Lakshman, 2019). Para los empleados que permanecen en la organización se han reportado cambios negativos en las condiciones de trabajo, al incrementarse la carga laboral, responsabilidades, aumento en los sentimientos de inseguridad laboral, rabia, estrés y una disminución en el compromiso y la lealtad (Andreeva, Brenner, Theorell, \& Goldberg, 2017; Andreeva, Magnusson Hanson, Westerlund, Theorell, \& Brenner, 2015; de Jong et al., 2016; Paterson \& Härtel, 2016), y para aquellos afectados directamente por la reducción de personal, la literatura científica se ha enfocado más en describir sus síntomas ansiosos y depresivos a causa de la pérdida de su trabajo (Andreeva et al., 2015; Snorradóttir, Tómasson, Vilhjálmsson, \& Rafnsdóttir, 2015).

En estas situaciones, la identidad grupal del trabajador tiene un papel fundamental. Existen tres aspectos que influyen en la percepción de justicia en las situaciones de recorte, 
despido o renegociación que son, en primer lugar, sí esto se debe a circunstancias externas o internas, sí los superiores tuvieron una actitud de solidaridad y sí los trabajadores despedidos fueron compensados adecuadamente (Charness \& Levine, 2000; Zwank \& Diehl, 2019). Las percepciones de justicia en momentos de despidos o recorte de personal son los predictores más fuerte de que las víctimas tomen la decisión de tomar alguna acción legal (Dutton J, 2017).

Para un manejo de los despidos acorde con una visión de justicia organizacional, Karl y Hancock, sugieren unos lineamientos de buenas prácticas: en primer lugar, se define que debe ser el supervisor inmediato quien notifique del despido, aunque puede estar presente también alguien externo, realizar la sesión en privado, también entrenar a los supervisores para ayudar al empleado a enfrentar el disconfort de la noticia (Karl \& Hancock, 1999).

\section{Castigos o amonestaciones}

El castigo dentro de una organización puede ser definido como la aplicación por parte del líder de una consecuencia negativa o el retiro de una consecuencia positiva a alguien bajo su supervisión, también se ha definido como una consecuencia negativa a determinado acto o comportamiento (Mooijman \& Graham, 2018; Trevino, 1992). La percepción de justicia para estos casos dependerá en gran medida en lograr una proporcionalidad entre la gravedad del acto de ruptura de la norma y el castigo impuesto a dicho trabajador, igualmente es necesario que la persona en posición de poder tenga claro cuál es el objetivo del castigo. Los trabajadores son especialmente sensibles a los castigos recibidos por ellos mismos y sus compañeros, en términos del nivel propio de castigo con respecto a la falta, en cuanto a su severidad y consistencia (Atwater, Waldman, Carey, \& Cartier, 2001; Ball, Trevino, \& Sims, 1994), y la percepción de justicia sobre el castigo se ha reportado que está mediada por la empatía que un trabajador tiene con quien está siendo castigado y con quien ejecuta el castigo (Lu \& McKeown, 2018; van Prooijen JW, 2015).

Entonces existen dos situaciones que pueden llevar a la percepción de injusticia acerca de los castigos o amonestaciones: por la consecuencia que generan, por el proceso previo de toma de decisiones que llevó al castigo (Mooijman \& Graham, 2018) y por el proceso comunicativo que se sigue durante todo el castigo (Andrighetto et al., 2016).

Cuando los castigos son percibidos como injustos por parte de los trabajadores, se puede generar el efecto conocido como miedo al castigo, FOP (fear of punishment) en inglés, El temor de un empleado por una amenaza de castigo puede estar relacionado con el grado en de percepción de que las reglas y procedimientos disciplinarios son injustos.

En un contexto percibido como justo, los procedimientos disciplinarios, a saber, las reglas del juego, deberían parecer estables y, por lo tanto, el entorno no debería parecer 
riesgoso para el empleado. Aunque las percepciones de la justicia procedimental pueden determinar principalmente la seguridad del entorno laboral, las percepciones desfavorables de la justicia interaccional pueden modificar la imagen original de la equidad de esos procedimientos (Goergen, Pauli, Cerutti, \& Perin, 2018).

\section{CONCLUSIÓN}

Con respecto a la pregunta formulada inicialmente, el panorama está abierto para que cada organización busque los medios necesarios para aumentar las percepciones de justicia de sus empleados. No obstante, esto lleva consigo un proceso de repensar las organizaciones, desde los aspectos básicos como la selección de personal hasta los procesos peor ponderados como los despidos o los recortes de personal.

Las percepciones de injusticia hacen que el desempeño y la satisfacción de los trabajadores sean menores y que estos busquen adoptar comportamientos contraproducentes como forma de retaliación frente a la injusticia, tales como robos, entorpecimiento de las tareas, mal manejo de los recursos y ausencias injustificadas de sus lugares de trabajo.

Desde el punto de vista positivo, la percepción de justicia contribuye a mantener la confianza y el respeto por las organizaciones, de manera tal, que, aunque sucedan acontecimientos organizacionales negativos, su impacto será amortiguado por esta percepción; principalmente cuando se hace un esfuerzo porque las percepciones de justicia procedimental e interaccional se mantengan altas.

El compromiso de los trabajadores también se ha reportado que depende en gran medida de la percepción de justicia que este tenga de su organización. De la misma manera, el talento excepcional será retenido con mayor facilidad.

Cada uno de los procedimientos que se llevan a cabo dentro de una organización conlleva el riesgo de generar una impresión de que se actúa injustamente o de que se desea beneficiar a algún individuo en particular. La justicia organizacional permite que estos procedimientos y decisiones se tomen de una manera más ecuánime, y aunque por sí sola no garantiza que todas las partes involucradas sientan que recibieron lo mejor posible, si hace que el poder sea manejado para el respeto de la dignidad de los trabajadores.

Desde el punto de vista investigativo, surge un amplio panorama de posibilidades en este tema. Es evidente la necesidad de contar con los resultados de estudios longitudinales que prueben cuales son las acciones sobre los procedimientos organizacionales que pueden aumentar la percepción de justicia de los trabajadores, igualmente es importante conocer cuál es el papel de las intervenciones comunicativas en el lugar de trabajo para tener una mayor visión de justicia, igualmente conocer el efecto de las intervenciones en el tema de la 
justicia organizacional sobre el compromiso laboral y los comportamientos contraproducentes en el trabajo.

Es necesario basarse en criterios éticos y actuar con miras siempre a lograr la equidad para lograr la máxima productividad y satisfacción de los trabajadores y la organización.

\section{REFERENCIAS}

Abas, N. A. H. b., Otto, K., \& Ramayah, T. (2018). A Supporting Hand in Dealing with Interpersonal Conflicts: The Role of Interactional Justice. Asian Academy of Management Journal, 23, 79-99. doi:10.21315/aamj2018.23.1.4

Adams, J. S. (1965). Inequity in Social Exchange. New York: Academic.

Allen, T., Whittaker, W., \& Sutton, M. (2017). Does the proportion of pay linked to performance affect the job satisfaction of general practitioners? Social Science \& Medicine, 173, 9-17. doi: https://doi.org/10.1016/j.socscimed.2016.11.028

Andreeva, E., Brenner, M. H., Theorell, T., \& Goldberg, M. (2017). Risk of psychological ill health and methods of organisational downsizing: A cross-sectional survey in four European countries. BMC Public Health, 17(1). doi:10.1186/s12889-017-4789-3

Andreeva, E., Magnusson Hanson, L. L., Westerlund, H., Theorell, T., \& Brenner, M. H. (2015). Depressive symptoms as a cause and effect of job loss in men and women: Evidence in the context of organisational downsizing from the Swedish Longitudinal Occupational Survey of Health. BMC Public Health, 15(1). doi:10.1186/s12889-015$2377-y$

Andrighetto, G., Brandts, J., Conte, R., Sabater-Mir, J., Solaz, H., Székely, Á., \& Villatoro, D. (2016). Counter-Punishment, Communication, and Cooperation among Partners. Frontiers in behavioral neuroscience, 10, 53-53. doi:10.3389/fnbeh.2016.00053

Atwater, L., Waldman, D., Carey, J., \& Cartier, P. (2001). Recipient and observer reactions to discipline: Are managers experiencing wishful thinking? Journal of Organizational Behavior, 22, 249-270. doi:10.1002/job.67

Babagana SA. (2019). Moderating Effect of Employee Participation on Factors that Determine Effective Performance Appraisal (EPA): Data Screening and Preliminary Analysis. International Journal of Academic Research Business and Social Sciences, 9(4), 116-134.

Ball, G. A., Trevino, L. K., \& Sims, H. P. (1994). Just and unjust punishment: Influences on subordinate performance and citizenship. Academy of Management Journal, 37(2), 299-322. doi:10.2307/256831

\footnotetext{
"Visión de Futuro" Año 17, Volumen No 24 N², Julio - Diciembre 2020 - Pág 166 - 183

URL de la Revista: http://visiondefuturo.fce.unam.edu.ar/index.php/visiondefuturo/index

URL del Documento: https://visiondefuturo.fce.unam.edu.ar/index.php/visiondefuturo/issue/view/18

ISSN 1668 - 8708 - Versión en Línea

E-mail: revistacientifica@fce.unam.edu.ar
} 
Bies, R. J., \& Moag, J. S. (1986). Interactional justice: Communication- criteria for fairness. In J. Press (Ed.), Research on Negotiation in Organizations (Vol. 1, pp. 43-55). Greenwich.

Blader, S. L., \& Tyler, T. R. (2009). Testing and extending the group engagement model: linkages between social identity, procedural justice, economic outcomes, and extrarole behavior. J Appl Psychol, 94(2), 445-464. doi:10.1037/a0013935

Booij, L. H. (2007). Conflicts in the operating theatre. Curr Opin Anaesthesiol, 20(2), 152156. doi:10.1097/ACO.0b013e32809f9506

Cameron, K. S., \& Freeman, S. (1996). Downsizing and redesigning organizations. In O. U. Press (Ed.), Organizational Change and Redesign: Ideas and Insights for Improving Performance (pp. 19-65). New York: Oxford University Press.

Cortini, M., Galanti, T., \& Barattucci, M. (2019). The Effect of Different Rejection Letters on Applicants' Reactions. Behavioral Sciences, 9(10), 102.

Cropanzano R \& Folger R. (1991). Procedural justice and worker motivation. In R. M. S. L. W. Porter (Ed.), Motivation and Work behavior (Vol. 5, pp. 131-143). New York: McGraw-Hill.

Cropanzano, R., \& Ambrose, M. (2001). Procedural and distributive justice are more similar than you think: A monistic perspective and a research agenda Advances in Organizational Justice (pp. 119-151). Stanford.

Cropanzano, R., Bowen, D. E., \& Gilliland, S. W. (2007). The management of organizational justice. The Academy of Management Perspectives, 21(4), 34-48. doi:10.5465/AMP.2007.27895338

Cropanzano, R., \& Mitchell, M. S. (2005). Social Exchange Theory: An Interdisciplinary Review. Journal of Management, 31(6), 874-900. doi:10.1177/0149206305279602 Charness, G., \& Levine, D. I. (2000). When are Layoffs Acceptable? Evidence from a QuasiExperiment. ILR Review, 53(3), 381-400. doi:10.1177/001979390005300302

de Jong, T., Wiezer, N., de Weerd, M., Nielsen, K., Mattila-Holappa, P., \& Mockałło, Z. (2016). The impact of restructuring on employee well-being: a systematic review of longitudinal studies. Work and Stress, 30(1), 91-114. doi:10.1080/02678373.2015.1136710

Denise Francis, L. (2019). Organizational justice, sensitivity to injustice and the experience of stress. Dissertation Abstracts International: Section B: The Sciences and Engineering, 64(1B), 451.

DeNisi, A. S., \& Murphy, K. R. (2017). Performance appraisal and performance management: 100 years of progress? J Appl Psychol, 102(3), 421-433. doi:10.1037/apl0000085

\footnotetext{
"Visión de Futuro" Año 17, Volumen N²4 N², Julio - Diciembre 2020 - Pág 166 - 183

URL de la Revista: http://visiondefuturo.fce.unam.edu.ar/index.php/visiondefuturo/index

URL del Documento: https://visiondefuturo.fce.unam.edu.ar/index.php/visiondefuturo/issue/view/18 
Dishon-Berkovits, M. (2017). The Role of Organizational Justice and Stress in Predicting Job Burnout. Journal of Career Development, 45(5), 411-424. doi:10.1177/0894845317705469

Dusterhoff, C., Cunningham, J., \& Macgregor, J. (2014). The Effects of Performance Rating, Leader-Member Exchange, Perceived Utility, and Organizational Justice on Performance Appraisal Satisfaction: Applying a Moral Judgment Perspective. Journal of Business Ethics, 119. doi:10.1007/s10551-013-1634-1

Dutton J. (2017). Positive Organizational Justice From Fair to Fairer-and Beyond Exploring Positive Relationships at Work Building a Theoretical and Research Foundation. New York: Lawrence Erlbaum Associates.

Fischer, R. (2008). Chapter 8: Organizational Justice and Reward Allocation (pp. 135).

Folger, R., \& Greenberg, J. (1985). Procedural justice: An interpretive analysis of personnel systems. Greenwich: JAI Press.

Gangloff, B., Mayoral, L., Personnaz, B., \& Rezazi, A. (2016). ¿Los Asesores de los Managers Son Conscientes de los Factores Determinantes en sus Sugerencias Sobre Distribución de Primas?: Un Estudio Sobre Justicia Distributiva. Psykhe (Santiago), 25, 1-13.

Giannantonio Cristina, M. (2019). Effects of recruiter friendliness and job attribute information on recruitment outcomes. Personnel Review, 48(6), 1491-1506. doi:10.1108/PR-012018-0037

Goergen, C., Pauli, J., Cerutti, P., \& Perin, M. (2018). The organizations. Justice, trust and retaliatory attitude: a study in a company the metal-mechanic sector. Cuadernos de Administración (Universidad del Valle), 34, 33-49.

Goldman, B. M. (2003). The Application of Referent Cognitions Theory to Legal-Claiming by Terminated Workers: The Role of Organizational Justice and Anger. Journal of Management, 29(5), 705-728. doi:10.1016/S0149-2063_03_00032-1

Greenberg. (1990). Organizational justice: Yesterday, today, and tomorrow. Journal of Management, 16(2), 399-432. doi:10.1177/014920639001600208

Greenberg. (1993). The social side of fairness: Interpersonal and informational classes of organizational justice. In R. Cropanzano (Ed.), Justice in the Workplace: Approaching Fairness in Human Resource Management In: (pp. 79-103). Hillsdale.

Greer, L. L., Saygi, O., Aaldering, H., \& de Dreu, C. K. (2012). Conflict in medical teams: opportunity or danger? Med Educ, 46(10), 935-942. doi:10.1111/j.13652923.2012.04321.x

Herr, R. M., Loerbroks, A., Bosch, J. A., Seegel, M., Schneider, M., \& Schmidt, B. (2016). Associations of Organizational Justice with Tinnitus and the Mediating Role of 
Depressive Symptoms and Burnout-Findings from a Cross-Sectional Study. Int J Behav Med, 23(2), 190-197.

Ibeogu, P. H., \& Ozturen, A. (2015). Perception of Justice in Performance Appraisal and Effect on Satisfaction: Empirical Findings from Northern Cyprus Banks. Procedia Economics and Finance, 23, 964-969. doi:https://doi.org/10.1016/S22125671(15)00359-7

Janss, R., Rispens, S., Segers, M., \& Jehn, K. A. (2012). What is happening under the surface? Power, conflict and the performance of medical teams. Medical Education, 46(9), 838-849. doi:10.1111/j.1365-2923.2012.04322.x

Karl, A., \& Hancock, B. (1999). Expert Advice on Employment Termination Practices: How Expert Is it? . Public Personnel Managendem, 28(1), 51-62.

Khiavi, F. F., Shakhi, K., Dehghani, R., \& Zahiri, M. (2016). The correlation between organizational justice and trust among employees of rehabilitation clinics in hospitals of Ahvaz, Iran. Electron Physician, 8(2), 1904-1910. doi:10.19082/1904

Konradt, U., Garbers, Y., Weber, M., Erdogan, B., \& Bauer, T. (2015). Antecedents and Consequences of Procedural Fairness Perceptions in Personnel Selection: A ThreeYear Longitudinal Study. Group and Organization Management, in press. doi:10.1177/1059601115617665

Lakshman, C. (2019). Consequences of paying CEOs for Downsizing: A Four-Country Study of the Impacts on Survivors vs. Victims. M@n@gement, 22(2), 250-272. doi:10.3917/mana.222.0250

Laundon, M., Cathcart, A., \& McDonald, P. (2019). Just benefits? Employee benefits and organisational justice. Employee Relations. doi:10.1108/ER-11-2017-0285

Leventhal, Karuza, \& Fry. (1980). Beyond Fairness A Theory of Allocation Preferences. Justice and Social Interaction Justice and Social Interaction (Vol. 3, pp. 167-218). New York: Springer-Verlag.

Loerbroks, A., Meng, H., Chen, M.-L., Herr, R., Angerer, P., \& Li, J. (2014). Primary school teachers in China: associations of organizational justice and effort-reward imbalance with burnout and intentions to leave the profession in a cross-sectional sample. Int Arch Occup Environ Health, 87(7), 695-703.

Long, C. P. (2016). Mapping the Main Roads to Fairness: Examining the Managerial Context of Fairness Promotion. Journal of Business Ethics, 137(4), 757-783. doi:10.1007/s10551-015-2749-3

Lu, T., \& McKeown, S. (2018). The effects of empathy, perceived injustice and group identity on altruistic preferences: Towards compensation or punishment. Journal of Applied Social Psychology, 48(12), 683-691. doi:10.1111/jasp.12558 
McCarthy, J., Bauer, T., Truxillo, D., Anderson, N., Costa, A. C., \& Ahmed, S. (2017). Applicant Perspectives During Selection A Review Addressing "So What?," "What's New?," and "Where to Next?". Journal of Management, 43, 1-33. doi:10.1177/0149206316681846

Meinecke, A. L., Lehmann-Willenbrock, N., \& Kauffeld, S. (2017). What happens during annual appraisal interviews? How leader-follower interactions unfold and impact interview outcomes. J Appl Psychol, 102(7), 1054-1074. doi:10.1037/apl0000219

Melchers, K. G., \& Körner, B. (2019). Is It Possible to Improve Test Takers' Perceptions of Ability Tests by Providing an Explanation? Journal of Personnel Psychology, 18(1), 1-9. doi:10.1027/1866-5888/a000212

Mooijman, M., \& Graham, J. (2018). Unjust punishment in organizations. Research in Organizational Behavior, 38, 95-106. doi:https://doi.org/10.1016/i.riob.2018.10.001

Mulders, J. O. (2019). Employers' age-related norms, stereotypes, and ageist preferences in employment. Innovation in Aging, 3(Suppl 1), S570-S570. doi:10.1093/geroni/igz038.2109

Nikolaou, I., \& Georgiou, K. (2018). Fairness reactions to the employment interview. Revista de Psicología del Trabajo y de las Organizaciones, 34, 103-111.

Paterson, J. M., \& Härtel, C. E. J. (2016). An integrated affective and cognitive model to explain employees' responses to downsizing Managing Emotions in the Workplace (pp. 25-44).

Pfeffer, J., \& Langton, N. (1993). The effect of wage dispersion on satisfaction, productivity, and working collaboratively: Evidence from college and university faculty. Administrative Science Quarterly, 38(3), 382-407. doi:10.2307/2393373

Rubin, E. V., \& Edwards, A. (2018). The performance of performance appraisal systems: understanding the linkage between appraisal structure and appraisal discrimination complaints. The International Journal of Human Resource Management, 1-20. doi:10.1080/09585192.2018.1424015

Sahoo, R. (2019). Organizational justice, conflict management and employee relations: The mediating role of climate of trust. International Journal of Manpower, 40(4), 783-799. doi:10.1108/IJM-12-2017-0342

Saxton, R. (2012). Communication skills training to address disruptive physician behavior. Aorn j, 95(5), 602-611. doi:10.1016/j.aorn.2011.06.011

Schulte-Braucks, J., Baethge, A., Dormann, C., \& Vahle-Hinz, T. (2019). Get even and feel good? Moderating effects of justice sensitivity and counterproductive work behavior on the relationship between illegitimate tasks and self-esteem. Journal of Occupational Health Psychology, 24(2), 241-255. doi:10.1037/ocp0000112 
Silva, M. R., \& Caetano, A. (2016). Organizational Justice Across Cultures: A Systematic Review of Four Decades of Research and Some Directions for the Future. Social Justice Research, 29(3), 257-287. doi:10.1007/s11211-016-0263-0

Snorradóttir, Á., Tómasson, K., Vilhjálmsson, R., \& Rafnsdóttir, G. L. (2015). The health and well-being of bankers following downsizing: A comparison of stayers and leavers. Work, Employment and Society, 29(5), 738-756. doi:10.1177/0950017014563106

Thomas, K., \& Kilmann, R. (1976). Thomas-Kilmann conflict MODE instrument. doi:10.1037/t02326-000

Trevino, L. K. (1992). The social effects of punishment in organizations: A justice perspective. The Academy of Management Review, 17(4), 647-676. doi:10.2307/258803

Truxillo, D. M., Bauer, T. N., \& Garcia, A. M. (2017). Applicant Reactions to Hiring Procedures The Wiley Blackwell Handbook of the Psychology of Recruitment, Selection and Employee Retention (pp. 53-70).

Tyler, T. R., \& Blader, S. L. (2000). Cooperation in groups: Procedural justice, social identity and behavioral engagement (Vol. 45). Philadelphia: Psychology Press.

Tziner, A., \& Levy, S. (2017). Examination of Performance Appraisal Behavior Structure. Frontiers in psychology, 7, 2075-2075. doi:10.3389/fpsyg.2016.02075

Umiker W. (2005). Management Skills for the New Health Care Supervisor (Vol. 3). Canada. Vaamonde, J. D., Omar, A., \& Salessi, S. (2018). From Organizational Justice Perceptions to Turnover Intentions: The Mediating Effects of Burnout and Job Satisfaction. Europe's journal of psychology, 14(3), 554-570. doi:10.5964/ejop.v14i3.1490

van den Bos, K., \& Lind, E. A. (2002). Uncertainty management by means of fairness judgments Advances in experimental social psychology, Vol. 34. (pp. 1-60). San Diego, CA, US: Academic Press.

van Prooijen JW. (2015). The punitive power holder: Social judgeability increases the severity of punishment. In IAP Information Age Publishing (Ed.), Research in Social Issues in Management. The social dynamics of organizational justice (pp. 121-140).

Wan, Q., Zhou, W., Li, Z., \& Shang, S. (2018). Associations of Organizational Justice and Job Characteristics with Work Engagement Among Nurses in Hospitals in China. Research in nursing \& health, 41(6), 555-562. doi:10.1002/nur.21908

Zwank, J., \& Diehl, M.-R. (2019). Fairness as a disguise: Fear-driven motives for justice enactment during organizational change. Academy of Management Proceedings, 2019(1), 16755. doi:10.5465/AMBPP.2019.245

\footnotetext{
"Visión de Futuro" Año 17, Volumen $N^{0} 24$ N², Julio - Diciembre 2020 - Pág 166 - 183

URL de la Revista: http://visiondefuturo.fce.unam.edu.ar/index.php/visiondefuturo/index

URL del Documento: https://visiondefuturo.fce.unam.edu.ar/index.php/visiondefuturo/issue/view/18

ISSN 1668 - 8708 - Versión en Línea

E-mail: revistacientifica@fce.unam.edu.ar
} 


\section{REFERENCIAS BIOGRÁFICAS}

\section{Elsa Maria Vasquez Trespalacios}

Bióloga, Magister en epidemiología, estudiante de Doctorado en Ciencias de la Salud ocupacional en la Universidad de Guadalajara, México. Actualmente docente e investigadora asociada, Universidad CES, Medellín, Colombia. Intereses de investigación: salud de los trabajadores, justicia de las organizaciones, estrés laboral.

\section{Carolina Aranda Beltran}

Médico cirujano y partero, Maestría en ciencias de la Salud Pública, especialidad en salud en el trabajo, doctorado en Ciencias de la Salud Pública. Actualmente profesora investigadora titular, Universidad de Guadalajara. Intereses de investigación: Salud de los trabajadores, Síndrome de Burnout. 